\title{
Design of a Small Automated Telescope for Indian Universities
}

\author{
Mandayam N. Anandaram, and B.A. Kagali \\ Department of Physics, Bangalore University, Bangalore 560 056, India. \\ e-mail:anandaram_mn@yahoo.com \\ S.P. Bhatnagar
}

Department of Physics, Bhavnagar University, Bhavnagar, India

\begin{abstract}
We have constructed a computer controlled telescope using a $0.36-\mathrm{m} \mathrm{f} / 11$ Celestron optical tube assembly for teaching and research applications. We have constructed a heavy duty fork-type equatorial mount fitted with precision machined 24 inch drive disks for both axes. These are friction driven by stepper motors through one inch rollers. We have used an open loop control system triggerable by an ST-4 CCD camera to acquire and track any target object. Our telescope can home in on any target within a range of two arc-minutes. We have employed a commercial stepper motor controller card for which we have written a user friendly pc based telescope control software in C. Photometry using a solid state photometer, and imaging by an ST-6 CCD camera are possible

We consider that this project is suitable for those wishing to construct some parts of a telescope and understand the principles of operation. A simpler model of this telescope could use DC motors instead of stepper motors. We shall be happy to send our design diagrams and details to those interested. This project was funded by the DST, and was assisted by IUCAA, Pune.
\end{abstract}

\section{Introduction}

We have undertaken the construction of the $0.36-\mathrm{m}$ automated photoelectric telescope (APT) having been inspired by similar developments abroad, and also facing the need of a small modern telescope for teaching of stellar photometry and carrying out minor research in our universities. At the suggestion of the instrumentation laboratory of IUCAA in Pune, we purchased the 0.36-m Schmidt-Cassegrain optical tube assembly made by Celestron of USA,stepper motors along with their digital driver units made by Aerotech of U.S.A., and the ST-4 and the ST-6 CCD cameras made by SBIG of U.S.A.. The telescope mount and other parts needed for assembling the telescope were locally fabricated with engineering design assistance of IUCAA. We now give a description of the relevant work done by us in India. A photo of the completed telescope mounted at Bhavnagar University sliding-roof observatory is displayed below. 


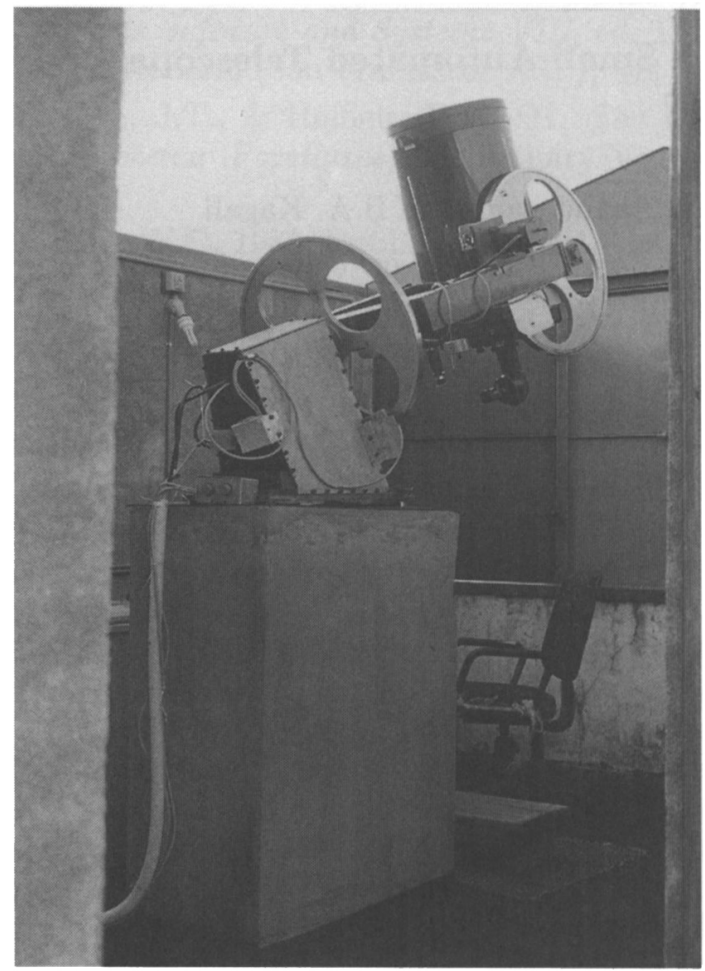

Figure 1. The 0.36-m APT Telescope

The telescope is illustrated in Figure 1 and a functional block diagram is shown in Figure 2.

\section{The Telescope Mount Design}

The telescope mount consists of a heavy-duty fork-type equatorial mount of rectangular cross-section. Two $0.6-\mathrm{m}$ drive disks with hardened chrome plated rims are attached to this fork. These disks are friction driven by one inch diameter rollers to produce RA and DEC axes rotations. The rollers are driven by the stepper motors which require 25,000 steps provided by their digital microstepper translator units. All the mechanical parts were fabricated in local workshops accoding to specifications listed in their detailed engineering diagrams. These parts were then carefully assembled together and tested. In our tests to measure the wobble of the rotating disks with a dial gauge, we found that the wobble was less than $0.1 \mathrm{~mm}$ for a complete rotation. This was considered adequate.

The RA base housing was first assembled with the 7.6- $\mathrm{cm}$ diameter RA shaft mounted on its bearings. Then the 0.6-m RA disk and the base of the fork were then mounted. One arm of the fork was then fitted with the DEC disk as well as the OTA mounting brackets. The $2.5-\mathrm{cm}$ rollers were then mounted under both disks such that they were in full contact with the smooth rims of 
the disks with adjustable pressure. The RA drive stepper motor was mounted behind the RA roller inside the RA axis cage and was dynamically coupled to it. Similarly the DEC drive motor was mounted on a fork arm and then coupled to its roller. Finally the OTA was mounted into the fork and weights on both fork arms were counter balanced.

The drive disks have been provided with rotation limiting stops inboth $\mathrm{CW}$ and CCW directions. The stoppers are so positioned that when the motion in RA axis is stopped the fork arms are horizontal and inclined at polar lattitude angle. Similarly when the motion in the DEC axis is stopped, the OTA is pointed towards the RA axis shaft. This position is defined as the PARK position of the telescope. To enable computer conrol three opto-coupled electronic position sensors have been provided for each axis. They provide logical high signals to identify the PARK position, the $\mathrm{CW}$ rotation limit just before its physical limit, and the CCW rotation limit just before its physical limit on both the $\mathrm{RA}$ axis and the DEC axis. When the limit signals are received the control program issues a command to stop the motion and/or reverse the direction of rotation as needed. Our control program utilises the PARK position signal to initialise the RA and DEC disk positions defined as the ZERO position. The DEC disk ZERO position is set to the value of -90 degree declination. The RA ZERO position is set at 180 degrees from the PARK position and from there the telescope can be driven to acquire any target object by software command. The occurrance of the $\mathrm{CW}$ limit signal allows the disk to commence rotation only in the CCW direction. Similarly an occurrance of the CCW limit signal allows a rotation only in the $\mathrm{CW}$ direction. The control program ensures that the telescope always begins its operation by sensing its current position and moves in the required direction if necessary to its PARK position. Then it would move from there to its ZERO position to initialise its position in memory so that the computer can continuously update the current position of both disks by the number of micro step pulses sent to both drive motors. In this way we have achieved an open loop control of the telescope.

\section{Control Program Design}

The 2.5 -cm roller coupled to the motor shaft rotates by $360^{\circ}$ on receiving 25,000 stepper pulses. Since the disk diameter is 24 times the roller diameter, we find that the telescope would rotate by one degree for 1666.7 stepper pulses. Thus each pulse causes an angular motion of 2.15 arc-second. This is the design pointing accuracy of our telescope and is used by the computer to determine the number of stepper pulses needed to cause the required angular motion in both axes to acquire the target.

We have employed a high speed programmable stepper motor controller card available in the market to generate pulses at the required rate for slewing the telescope rapidly and also to enable it to track any star after acquiring it. This card is installed in a personal computer and its input/output lines are connected to the digital microstepper controller boxes attached to the motors as well as the outputs of position sensors on the drive disks. Provision exists to add additional control lines from instruments like photometers and CCD cameras. 


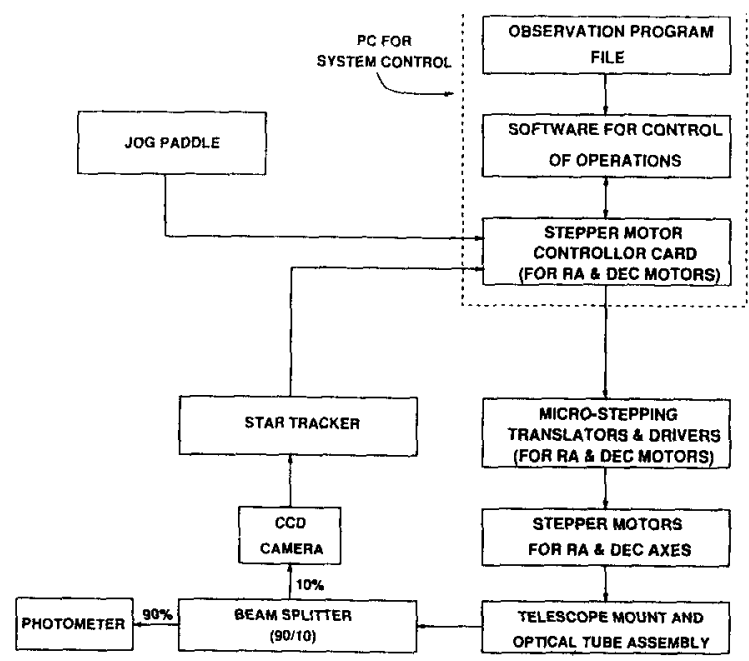

FUNCTIONAL FLOWCHART OF THE APT

Figure 2. Block Diagram of the Telescope

We have written a user friendly telescope control program in $\mathrm{C}$ which offers a menu based choice of operations.

\section{Performance of the Telescope}

We have carried out several types of performance checks on the operation of our telescope. In one test for the reproducibility of a specified pointing direction, the telescope was moved in RA or DEC axes by $50^{\circ}$ and then moved back to the original direction by program command. We found that the slippage in pointing is 2 arc-minutes in $50^{\circ}$, or,just 2.4 arc-second per degree. This was considered satisfactory. We find that our telescope has a satisfactory target acquisition ability of about 2 arc-minutes in its field of view.

This telescope is presently equipped with a solid state UBV photometer for carrying out photometry, a ST-4 CCD used as a star tracker, and a ST-6 CCD camera for use in imaging applications. We plan to convert the telescope control from open loop to closed-loop control by adding digital position encoders.

In summary, we find that building this telescope has been a challenging and educative experience. We shall be happy to send our detailed design diagrams and helpful advice to any one interested in building a similar telescope.

Acknowledgments. This project was made possible by DST project grants to both of us (M.N.A and S.P.B), and extensive assistence rendered by IUCAA in Pune, India. 